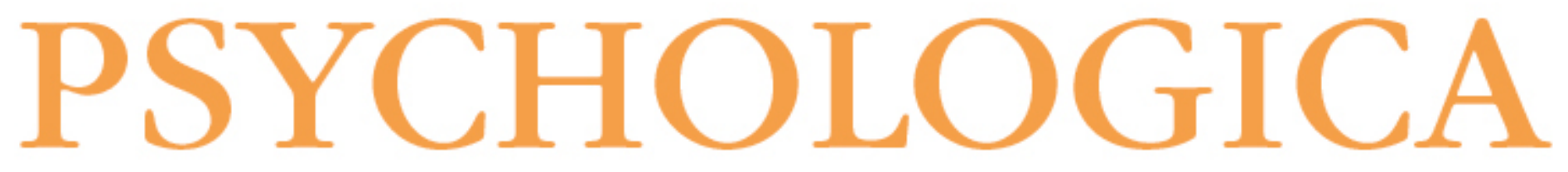

Avaliação de estímulos emocionais em ex-combatentes de guerra com e sem PTSD

Autor(es): $\quad$ Pinto, Carla; Esteves, Francisco

Publicado por: Imprensa da Universidade de Coimbra

URL

persistente: URI:http://hdl.handle.net/10316.2/5465

DOI: $\quad$ DOI:http://dx.doi.org/10.14195/1647-8606_51_13

Accessed : $\quad$ 26-Apr-2023 12:09:48

A navegação consulta e descarregamento dos títulos inseridos nas Bibliotecas Digitais UC Digitalis, UC Pombalina e UC Impactum, pressupõem a aceitação plena e sem reservas dos Termos e Condições de Uso destas Bibliotecas Digitais, disponíveis em https://digitalis.uc.pt/pt-pt/termos.

Conforme exposto nos referidos Termos e Condições de Uso, o descarregamento de títulos de acesso restrito requer uma licença válida de autorização devendo o utilizador aceder ao(s) documento(s) a partir de um endereço de IP da instituição detentora da supramencionada licença.

Ao utilizador é apenas permitido o descarregamento para uso pessoal, pelo que o emprego do(s) título(s) descarregado(s) para outro fim, designadamente comercial, carece de autorização do respetivo autor ou editor da obra.

Na medida em que todas as obras da UC Digitalis se encontram protegidas pelo Código do Direito de Autor e Direitos Conexos e demais legislação aplicável, toda a cópia, parcial ou total, deste documento, nos casos em que é legalmente admitida, deverá conter ou fazer-se acompanhar por este aviso. 
NÚMERO 51

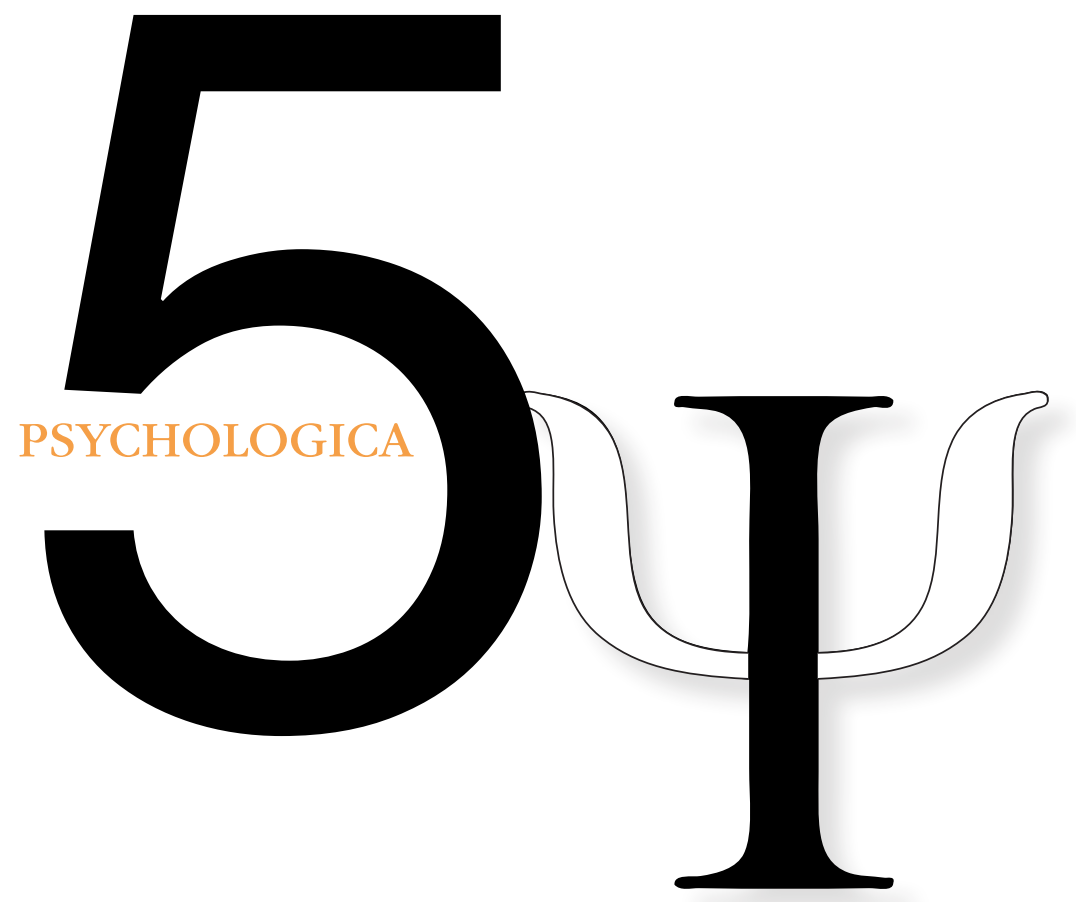

IMPRENSA DA UNIVERSIDADE DE COIMBRA

FACULDADE DE PSICOLOGIA E DE CIÊNCIAS DA EDUCAÇÃO DA UNIVERSIDADE DE COIMBRA 


\title{
Avaliação de estímulos emocionais em Ex-Combaten- tes de Guerra com e sem PTSD
}

\author{
Carla Pinto' e Francisco Esteves ${ }^{2}$
}

O presente estudo teve por objectivo averiguar a forma como são avaliadas imagens emocionais por Ex-Combatentes de Guerra. O estudo foi realizado com uma amostra de 72 sujeitos que foram submetidos a duas escalas de avaliação de PTSD, e à visualização e categorização das imagens através do Self-Assessement Manikin (SAM; Lang, Bradley \& Cuthbert, 1997).

Foram comparados três grupos, um com diagnóstico de PTSD (Grupo PTSD) e dois sem diagnóstico de PTSD, constituídos com base nas escalas de auto-avaliação: um com valores baixos (Grupo Não-Clínico Baixo - NCB) e outro com valores mais elevados (Grupo Não-Clínico Alto - NCA). Verificou-se que tanto o grupo com PTSD como o grupo NCA avaliam mais negativamente as imagens desagradáveis (imagens de guerra e "caras zangadas") em comparação com o grupo NCB. Além disso, globalmente, os mesmos grupos também avaliam as imagens desagradáveis como mais activadoras e dominantes que o grupo NCB. Em relação às imagens agradáveis, a diferenciação entre os três grupos não foi significativa.

PALAVRAS-CHAVE: Emoção; PTSD; Entorpecimento emocional; Combatentes de guerra.

A Perturbação Pós-Stress Traumático foi originalmente conceptualizada como uma consequência directa da exposição a um episódio traumático, superior à experiência humana considerada "normal" (Halligan \& Yehuda, 2000). Ou seja, "é uma consequência persistente, normativa e primária" (Engdahe, Dikel, Ebery \& Blank, 1997, p.1578) que decorre da exposição a um stressor traumático extremo ao qual a pessoa responde com intenso medo, desespero ou horror. Os sintomas característicos da exposição ao trauma incluem: reexperienciar persistente do episódio traumático através de pensamentos, pesadelos e imagens perturbadoras, evitamento persistente dos estímulos associados ao acontecimento traumático

\footnotetext{
1 Departamento de Psicologia, Universidade Lusófona de Humanidades e Tecnologias. Assistente de Saúde - Área de Psicologia - na Unidade Comunitária de Cuidados Psiquiátricos de Odivelas (Equipa A do Pólo Júlio de Matos do Centro Hospitalar Psiquiátrico de Lisboa).

2 Professor Auxiliar no Departamento de Psicologia Social e das Organizações do Instituto Superior de Ciências do Trabalho e da Empresa.
} 
e embotamento geral da reactividade, aumento dos sintomas de irritabilidade, de activação, sobressalto, distúrbios do sono (APA, 2002), sentimentos de culpa de sobrevivência, abuso de álcool ou drogas (Goodwin, 1987; Albuquerque et al., 1992), perda de crenças, ódio próprio, diminuição na capacidade para sentir prazer, satisfação e alegria (van der Kolk, 2001), distanciamento interpessoal (Keane, 1993), perturbações de memória (Golier, Yehuda \& Southwick, 1997; Buckley, Blanchard, Neill, 2000) e diminuição na regulação do afecto (Anunciação, 2004; van der Kolk, 2001; Friedman, 1997). A sintomatologia provoca mal-estar ou é incapacitante para a vida laboral, familiar e social (Anunciação, 2004).

Segundo Friedman e Yehuda (1995), "a PTSD como entidade clínica raramente ocorre numa forma pura" (Friedman \& Yehuda, 1995, p.429). É, por norma, acompanhada por outras perturbações psicológicas (Friedman \& Yehuda, 1995; Foa, Keane \& Friedman, 2000), tal como depressão, somatização e perturbações dissociativas (Friedman, 1997).

A PTSD engloba inúmeras emoções, como por exemplo a ansiedade e o medo, e é provocada por intensas experiências emocionais (Litz, Orsillos, Koloupek \& Weathers, 2000). Representa um mecanismo de resposta dirigido para a resolução de problemas de adaptação e tem componentes de cariz automático, sendo frequentemente desencadeada fora do limiar da consciência. Este tipo de emoções têm como objectivo primordial promover a sobrevivência de um organismo (Damásio, 2001).

O reconhecimento da importância das reacções emocionais tem vindo a ocupar uma posição central no campo da psicologia e, mais especificamente, no campo das perturbações relacionadas com eventos traumáticos. As recentes pesquisas são quase tão vastas e diversas como a vida emocional em si (Cacioppo e Gardner, 1999). Todavia, até há bem pouco tempo a investigação psicológica havia negligenciado a sua importância no campo da PTSD. A experiência do trauma produz emoções arrebatadoras que são a característica central das consequências psicológicas de longo prazo dos acontecimentos traumáticos (Litz et al., 2000), como o medo esmagador, o horror e a ansiedade. Estas acções podem manifestarse durante toda a vida, uma vez que "imagens mentais intrusivas podem surgir espontaneamente na mente, acompanhadas de elevados níveis emocionais" (Holmes, 2003/2004, p.147). Muitos sobreviventes de traumas referem, também, restrições nas suas experiências emocionais e, algumas vezes, embotamento em situações em que é suposto expressar e experienciar emoções. Este fenómeno é comummente referido como entorpecimento emocional (Litz \& Gray, 2002). Ou seja, o entorpecimento emocional pode ser visto como um défice generalizado em produzir respostas emocionais ou como um défice emocional nas respostas a eventos positivos (Litz, 1992). Litz (1992) propõe que o entorpecimento emocional 
é um complexo determinado por múltiplas variáveis, caracterizado por um défice no processo selectivo emocional. Todavia, "o entorpecimento emocional tem sido alvo de uma grande confusão na literatura clínica” (Litz et al., 2000, p.26). Este fenómeno emocional tem sido conceptualizado como uma defesa de emoções esmagadoras (Foa \& Hearst-lkeda, 1996) e como uma forma de evitamento (Keane, Street \& Orcutt, 2000).

Foa e Hearst-Ikeda (1996) defendem que a inclusão dos sintomas de evitamento e entorpecimento num mesmo critério leva a crer que os autores do DSM-IV conceptualizam o entorpecimento emocional e o esforço de evitar como conceitos equivalentes. No entanto, na revisão de literatura feita por estes autores, é possível verificar que o entorpecimento e o evitamento envolvem mecanismos diferentes. O evitamento é regulado por processos psicológicos estratégicos, enquanto que o entorpecimento pode ser mediado por mecanismos biológicos semelhantes aos do comportamento de congelamento ("freeze") em animais assustados. Os autores propõem que numa exposição a informação relacionada com o trauma, as vítimas mobilizam, primeiramente, esforços para evitar a activação associada às memórias traumáticas, e que quando estas estratégias falham ocorre um "desligamento" dos sistemas afectivos (Foa \& Hearst-Ikeda, 1996).

Segundo Litz (1992), possíveis explicações para o entorpecimento, ou perda da resposta emocional que algumas pessoas diagnosticadas com PTSD referem, são: a) os sintomas de entorpecimento resultam de um evitamento crónico de estímulos que doutra maneira provocariam uma série de reacções emocionais; b) o estímulo emocional pode estar disponível, sendo contudo de difícil acesso; c) estímulos emocionais podem ser percebidos, mas a resposta emocional é inibida e/ou impedida; ou d) as pessoas diagnosticadas com PTSD gastam as suas energias com as estratégias de coping para lidar com a reexperienciação, e com os sintomas de hiper-activação, saturando os seus recursos afectivos (Litz, 1992, p.418). Na tentativa de perceber melhor a relação entre hiperemotividade e os subsequentes défices no processamento emocional na PTSD, Litz e colaboradores (2000) expuseram dois grupos de ex-combatentes de guerra do Vietname (com e sem PTSD) a um vídeo evocador do trauma (em comparação com um vídeo de conteúdo neutro). Depois de observarem os vídeos, os participantes eram expostos a fotografias de conteúdo positivo, negativo e neutro. As respostas emocionais foram avaliadas através da actividade autónoma periférica, respostas expressivas motoras e através da auto-avaliação das reacções emocionais. Os resultados obtidos remetem para uma modulação comportamental emocional de ambos os grupos para as imagens de conteúdo neutro. Quanto às imagens de conteúdo positivo, o grupo com PTSD exibe uma supressão nas respostas expressivas motoras em comparação com o grupo de controlo, e, contrariamente ao que se esperava, o grupo com PTSD não apresentam aumentos significativos das respostas emocionais a 
estímulos negativos. No entanto verificou-se que o grupo com PTSD responde a todas as imagens com resposta cardíaca relativamente elevada, o que, segundo os autores, sugere uma preparação automática para a ameaça em qualquer contexto emocional impreciso. Assim, Litz e colaboradores (2000) concluíram que o entorpecimento é melhor explicado como um défice no processamento emocional, tendo em conta os episódios de hiperemotividade que são desencadeados pela exposição a memórias do acontecimento traumático, e como uma diminuição na capacidade para experimentarem sentimentos positivos e de serem mais reactivos para padrões negativos.

Goodwin (1987) caracteriza o entorpecimento emocional como um mecanismo de sobrevivência dinâmico que ajuda o indivíduo a passar por um período de trauma sem ser envolvido pelas suas armadilhas mentais. $O$ autor relata que alguns veteranos entrevistados referem que "se se permitirem sentir uma só vez, podem não mais parar de chorar ou podem perder o controlo sobre eles próprios. Todavia, muitos dos veteranos de guerra têm uma capacidade ímpar de amar e cuidar dos outros. Ainda assim, alguns deles não sabem qual o sentido da vida nem o porquê de existirem" (Goodwin, 1987, p.12). Contudo, Litz e Gray (2002), referem que não vêm razão para afirmar de forma assertiva que PTSD implica uma incapacidade para aceder a qualquer estado emocional disponivel no pré-trauma. O que os autores defendem é que os indivíduos que passaram por um evento traumático requerem uma maior estimulação para acederem a emoções de valência positiva e que, em qualquer contexto, estão dispostos a responder a circunstâncias emocionais negativas, ainda que de um nível de intensidade mais baixo (Litz e Gray, 2002).

No presente estudo pretende verificar-se de que forma são avaliados estímulos visuais emocionais (imagens de guerra, imagens de conteúdo positivo e rostos humanos com diferentes expressões emocionais), por Ex-Combatentes de Guerra. Comparando três grupos, um com diagnóstico de PTSD, e dois de participantes sem diagnóstico de PTSD, constituídos com base em escalas de auto-avaliação: um com valores baixos e outro com valores mais elevados, o que se espera observar com a apresentação destas imagens emocionais é: (a) diferenças na avaliação emocional das imagens mediante o sujeito tenha ou não PTSD, ou seja, que os sujeitos com PTSD diagnosticado, ou com valores mais elevados de PTSD em escalas de auto-relato, avaliem as imagens de guerra e as caras ameaçadoras de forma mais negativa; (b) maior dificuldade em avaliar de forma favorável imagens de conteúdo positivo em sujeitos com PTSD diagnosticado, ou com níveis de PTSD mais elevados nas escalas de auto-avaliação, por comparação com o grupo sem PTSD (c.f. Litz et al., 2000). 


\section{Método}

\section{Participantes}

Participaram neste estudo setenta e dois sujeitos, todos do sexo masculino, Ex-Combatentes de Guerra com e sem deficiência e com e sem PTSD. Uma vez que no âmbito do estudo foram mostradas imagens de guerra, os sujeitos que preencheram o critério dois do DSM-IV foram excluídos do estudo, ou seja, aqueles que evitam de forma sistemática os estímulos associados com o trauma e/ou o embotamento da reactividade geral. Foi excluído um participante por preencher este critério.

A idade média dos participantes é de 58,5, com um DP $=4,6$. Relativamente ao estado civil, $87,7 \%$ dos participantes são casados, $2,7 \%$ solteiros, $8,2 \%$ divorciados ou viúvos. As respectivas profissões estão representadas na Tabela 1. Quanto ao nivel de escolaridade, $63 \%$ estudou até à quarta classe e $35,6 \%$ mais que a quarta classe. No que respeita ao meio em que vivem, $46,6 \%$ vive no meio rural, $21,9 \%$ num meio Suburbano e 30,1\% num meio Urbano.

Tabela 1. Caracterização dos dados demográficos da amostra.

\begin{tabular}{lcc}
\hline Idade & Média & DP \\
& 58,5 & 4,6 \\
& $\mathrm{~N}$ & $\%$ \\
\hline Escolaridade & & \\
$\quad$ Até à $4^{\text {a }}$ Classe & 46 & $63,0 \%$ \\
$\quad$ Mais que ${ }^{\text {a }}$ Classe & 26 & $35,6 \%$ \\
\hline Meio & & \\
$\quad$ Rural & 34 & $46,6 \%$ \\
$\quad$ Suburbano & 16 & $21,9 \%$ \\
$\quad$ Urbano & 22 & $30,1 \%$ \\
\hline Estado civil actual & & \\
Casado & 64 & $87,7 \%$ \\
$\quad$ Solteiro & 2 & $2,7 \%$ \\
$\quad$ Divorciado & 3 & $4,1 \%$ \\
$\quad$ Viúvo & 3 & $4,1 \%$ \\
\hline Profissão actual & & \\
Reformado & 38 & 52,1 \\
Desempregado & 5 & 6,8 \\
Activo & 29 & 38,5 \\
\hline
\end{tabular}

Em relação às variáveis referentes à guerra, nomeadamente no que se refere ao posto ocupado pelos indivíduos, os resultados remetem para 75,3\% de Praças, $16,4 \%$ de Sargentos/Furriéis e 6,8\% de Oficiais. Trinta e sete estiveram em Angola, dezasseis em Moçambique, catorze na Guiné, e quatro estiveram em dois sítios (ver Tabela 2). O tempo de permanência varia entre 4 e 78 meses, sendo a média 25,2 meses, com um DP de 15,6. Quanto à idade com que foram para a guerra, esta varia entre 18 anos e 30 anos, com uma média de 21,35 e DP de 1,7. Quando 
os sujeitos partiram para a guerra eram, na sua grande maioria, solteiros $(86,1 \%)$ e apenas uma percentagem menor era casada $(13,9 \%)$.

Tabela 2. Caracterização dos dados de Guerra.

214

\begin{tabular}{lcc}
\hline & $\mathbf{N}$ & $\%$ \\
\hline Posto & 55 & $76,4 \%$ \\
Praças & 12 & $16,7 \%$ \\
Sargentos/Furriéis & 5 & $6,9 \%$ \\
Oficiais & & \\
\hline Local de combate & 37 & $50,7 \%$ \\
Angola & 16 & $21,9 \%$ \\
Moçambique & 14 & $19,2 \%$ \\
Guiné & 1 & $1,4 \%$ \\
Moçambique e Angola & 1 & $1,4 \%$ \\
Cabo Verde & 2 & $2,7 \%$ \\
Guiné e Angola & 1 & $1,4 \%$ \\
Índia & Média & DP \\
\hline Tempo de permanência & 25,18 & 15,6 \\
\hline Idade de ida para guerra & 21,4 & 1,7 \\
\hline & $\mathrm{N}$ & $\%$ \\
\hline Estado civil antes da guerra & 10 & $13,9 \%$ \\
Casado & 62 & $86,1 \%$ \\
\hline Solteiro & & \\
\hline
\end{tabular}

\section{Instrumentos}

Com o intuito de avaliar a PTSD foi pedido aos sujeitos que respondessem a dois questionários de auto-avaliação: a The Impact of Event Scale (IES; Horowitz, Wilner, Alvarez, 1979), utilizada na consulta de Psicologia de PTSD do Hospital Júlio de Matos (traduzida por Albuquerque e Lopes) e a The PTSD Checklist (PCL; Weathers, Litz, Herman, Huska \& Keane; 1993, traduzida pelos autores do presente estudo).

A IES é constituída por quinze itens, sete avaliam sintomas intrusivos (pensamentos intrusivos, pesadelos, sentimentos ou imagens intrusivas) e os restantes oito avaliam a evitação dos sintomas (evitação de situações, sentimentos e ideias) que, em conjunto, resultam num valor de stress subjectivo total. Esta escala tem um formato de resposta tipo Likert de quatro níveis, a saber: o (nunca), 1 (raramente), 3 (algumas vezes) e 5 (muitas vezes).

No que respeita à amplitude dos resultados, os valores da sub-escala "Fenómenos Intrusivos" variam entre o e 35 e resultam da soma dos valores dos itens 1,4,5,6,10,11 e 14. Os resultados da "Evitação" variam entre o e 40 e corresponde à soma dos itens $2,3,7,8,9,12,13$ e 15 . A soma das duas sub-escalas corresponde ao valor total de 
stress. Valores totais acima de 26 são indicadores de stress, ou seja, são indicadores de uma perturbação moderada ou grave de stress traumático (Horowitz et al.,1979).

No que concerne à consistência interna das sub-escalas, os valores apurados, baseados numa amostra de dois grupos distintos, são reveladores de uma consistência interna elevada. Os coeficientes variam entre 0,79 e 0,92, com um valor médio de 0,86 para a escala "Fenómenos Intrusivos" e o,90 para a escala "Evitação". O valor de correlação de o,42 entre as sub-escalas indica que estão associadas, mas não avaliam dimensões semelhantes. A escala IES tem uma elevada fidelidade $(r=0,86)$ e estabilidade temporal, uma vez que o valor de teste-reteste é de 0,87 para a escala de stress total, de 0,89 para a sub-escala Intrusão e de 0,79 para a subescala Evitação. A validade da escala foi verificada através da aplicação da mesma a um grupo de 32 sujeitos, antes, e onze semanas depois, de serem submetidos a tratamento da PTSD. Os resultados obtidos confirmaram uma diminuição nos valores obtidos na escala total (Horowitz et al., 1979).

A PCL, por sua vez, consiste em 17 itens que correspondem aos sintomas de PTSD do DSM-IV. A escala tem um formato de resposta tipo Likert de 5 pontos, em que 1 corresponde a "Não de todo" e 5 a "Extremamente" (Weathers et al., 1993). Existem duas versões desta escala: a PCL-M, que avalia o reviver de sintomas específicos de experiências militares, e a PCL-C, que avalia o reviver de sintomas em qualquer situação traumática. Devido à nossa amostra ser constituída apenas por casos de guerra, foi aplicada a PCL-M.

Foram encontrados valores de consistência interna de 0,93 para os sintomas B do DSM, 0,92 para sintomas $C, 0,92$ para sintomas $D$ e o,97 para os 17 sintomas. Os valores de correlação inter-itens da escala total variam entre o,62 e o,87 (Weathers, et al., 1993). A estabilidade temporal apresenta um valor de teste-reteste de o,96, revelando-se, desta forma, muito elevada. A validade convergente foi obtida através da forte correlação entre a PCL e: a Mississipi Scale $(0,93)$, a escala PK do MMPI-2 (o,77), a The Impact of Event Scale $(0,90)$ e a Combat Exposure Scale $(0,46)$ (Weathers, et al., 1993).

Para a apresentação dos estímulos, foi utilizado um computador com o programa PowerPoint. As imagens apresentadas foram seleccionadas da seguinte forma: Dez imagens de Expressões faciais (cinco agradáveis e cinco ameaçadoras), foram seleccionadas do Karolinska Directed Emotional Faces (KDEF, Lundqvist, Flykt \& Öhman., 1998). O KDEF consiste num conjunto de fotografias de actores, representando diferentes expressões faciais emocionais (feliz, surpreso, medo, repugnância, tristeza, raiva e neutras) de acordo com as normas apresentadas por Ekman and Friesen (1975). As caras ameaçadoras correspondem às expressões que pretendem representar a raiva, as agradáveis correspondem às felizes. As dez imagens de Guerra foram inicialmente seleccionadas do International Affective Picture System (IAPS; Lang, Bradley \& Cuthbert, 1997), mas por não existirem 
muitas de acordo com os nossos objectivos, foi necessário acrescentar outras seleccionadas através da internet.

Foi também utilizado um questionário de dados demográficos (idade, escolaridade, profissão, meio em que vive, onde participou na guerra, posto militar, tempo de permanência na guerra, estado civil antes de ir para a guerra, idade quando partiu para a guerra e profissão antes de participar na guerra).

Por forma a avaliar cada imagem, foi utilizado o Self-Assessment Manikin (SAM; Lang, 1980). O SAM consiste num método rápido e eficaz para a avaliação subjectiva do impacto emocional de um estímulo (Lang et al., 1997). Foram avaliadas três dimensões: Valência Afectiva (prazer/desprazer), Activação (calmo/excitado) e a Dominância (domínio da situação/dominado pela situação). A dimensão Valência Afectiva é composta por cinco figuras que variam entre uma expressão feliz e sorridente e uma expressão muito infeliz. A dimensão Activação é, também, composta por um extremo que representa uma expressão relaxada/dormir sem activação, e um outro pólo que representa uma figura com uma grande excitação/ agitação. A dimensão Dominância varia entre um pólo representado por uma figura de tamanho muito inferir ao ponto médio e uma figura com tamanho muito superior ao ponto médio. Cada uma das dimensões é composta por cinco figuras o que, contanto com a possibilidade de assinalar entre figuras, resulta numa escala de nove pontos.

A validade do SAM, por comparação com a Escala Semântica Diferencial elaborada por Mehrabian e Russel, resultou numa elevada correlação entre as classificações de prazer e activação de ambas. A avaliação das médias da Valência Afectiva e da Activação apresentaram uma consistência interna elevada testada pelo método de split-half $(0,94)$. A análise do teste-reteste revelou valores de 0,99 e 0,97 para as dimensões Valência Afectiva e Activação, respectivamente (Lang et al., 1997).

Assim, o protocolo era constituído por uma página de rosto onde se apresentava o estudo, era garantida a confidencialidade do mesmo, a participação voluntária e a possibilidade de desistir, seguida de vinte e duas representações do SAM para avaliar as imagens projectadas no PowerPoint do computador e, por fim, duas escalas para avaliar a PTSD.

\section{Procedimento}

Os sujeitos foram contactados previamente afim de lhes ser explicado o conteúdo do trabalho para que, desta forma, pudessem ponderar a sua participação ou não, no estudo. Depois do seu consentimento, procedeu-se à marcação do local dia e hora para a realização do estudo. 
Os participantes, ao entrar na sala, eram convidados a sentar-se numa cadeira, que Ihes havia sido previamente destinada. Seguiu-se a instrução acerca do conteúdo da experiência e seus objectivos. O estudo, em si, consistiu: em primeiro lugar na recolha de alguns dados demográficos; em segundo lugar em mostrar imagens em PowerPoint ao sujeito, antecipadas por um ponto, no qual os participantes deveriam fixar a sua atenção durante um segundo. Depois das imagens desaparecerem do campo de visão seguia-se uma tarefa de avaliação para cada uma delas, através das três valências do SAM, previamente explicada. A ordem das imagens foi diferente para diferentes participantes (quatro ordens de apresentação diferentes). O tempo de exposição de cada imagem foi de seis segundos, antecipada por um sinal que alerta para a apresentação da imagem (um ponto no ecrã do computador) e seguida de um período de avaliação da mesma (ecrã branco). As duas primeiras imagens serviram de treino. Em seguida foram passadas duas escalas de avaliação da PTSD (IES e PCL-M), com prévia explicação das mesmas, apesar das escalas possuírem instruções no cabeçalho. Por fim, foi perguntado verbalmente ao sujeito se estava diagnosticado com PTSD e se estava a receber tratamento para a mesma.

A informação recolhida foi introduzida em base de dados em Excel e, posteriormente trabalhada no programa SPSS.

\section{Resultados}

Para proceder à comparação entre os participantes com e sem PTSD, foram formados três grupos: um constituído por sujeitos sem diagnóstico de PTSD e sem valores significativos de patologia em qualquer das escalas PCL-M e IES (Grupo Não-Clínico Baixo - NCB), um segundo grupo sem diagnóstico de PTSD mas com valores significativos de patologia nas escalas (Grupo Não-Clínico Alto - NCA) e um terceiro com diagnóstico de PTSD (Grupo PTSD). Todavia, o número de indivíduos em cada grupo varia consoante a escala, sendo que, tendo como referência a escala PCL-M, os grupos são constituídos por 24 sujeitos cada, tendo por base a IES, o Grupo NCB é formado por 27 sujeitos, o Grupo NCA por 21 e o Grupo PTSD por 24.

Com o objectivo de verificar se existiam diferenças na avaliação dos estímulos em função do Grupo, a análise dos dados foi feita separadamente para cada uma das dimensões do SAM. Foram efectuadas análises de variância (ANOVAs) $3 \times 4$ para cada uma das dimensões, com a variável Grupo com medida inter-sujeito, e Categoria de Imagens (caras alegres, caras zangadas, desporto e guerra) como variável intra-sujeito. 
Tabela 3. Grupos em função das Escalas IES e PCL-M.

\begin{tabular}{llll}
\hline & N & Média & DP \\
\hline IES & & & \\
Grupo NCB & 27 & 11,89 & 6,76 \\
Grupo NCA & 21 & 45,48 & 14,67 \\
Grupo PTSD & 24 & 54,21 & 11,67 \\
\hline PCL-M & & & \\
Grupo NCB & 24 & 21,75 & 3,22 \\
Grupo NCA & 24 & 43,79 & 14,94 \\
Grupo PTSD & 24 & 62,33 & 13,90 \\
\hline
\end{tabular}

Legenda: Grupo NCB - grupo não-clínico baixo; Grupo NCA - grupo não-clínico alto; Grupo PTSD - grupo clínico com diagnóstico de PTSD; IES - Impact of Event Scale; PCL-M - PTSD Checklist

\section{Comparação das avaliações das imagens emocionais, entre os três gru- pos constituídos através da PCL-M}

No que se refere aos resultados obtidos na dimensão Valência do SAM, apurou-se um efeito principal do Grupo, $F(2,69)=3,32$, p<0,05. Uma análise post-hoc de Tukey revelou que o Grupo NCB fez avaliações mais positivas das imagens do que o Grupo PTSD. Também se verificou um efeito principal da Categoria, $\mathrm{F}(3,207)$ = 301,57, p<0,001. Como se pode ver no Gráfico 1, as imagens agradáveis (caras alegres e desporto) foram avaliadas mais positivamente que as imagens desagradáveis (caras zangadas e imagens de guerra). Finalmente também a interacção entre Grupo e Categoria foi significativa, $F(6,207)=4,11, p<0,001$. Para analisar em que imagens se verificavam diferenças entre os grupos, foram feitas análises univariadas para cada categoria das imagens. Verificou-se que apenas existiam diferenças entre os grupos nas caras zangadas, $F(2,72)=9,12, p<0,001$, e nas imagens de guerra, $F(2,72)=3,15, p<0,05$. De uma forma geral, o Grupo NCB faz uma avaliação destes estímulos mais positiva que os Grupos NCA e PTSD (ver Gráfico 1). Testes de Tukey revelaram que as diferenças entre o Grupo NCB e os outros dois grupos são significativas para as caras zangadas, mas em relação às imagens de guerra, apenas foi significativa a diferença entre o Grupo NCB e o Grupo NCA.

No que respeita aos resultados obtidos na dimensão Activação do SAM, os valores mostram que existe um efeito principal do Grupo, $F(2,69)=4,77, p<0,05$. Uma análise post-hoc de Tukey revelou que o Grupo PTSD avalia a sua activação como mais forte que o Grupo NCB. Também se verificou um efeito principal da Categoria, $F(3,207)=162,00, p<0,001$. Tal como é possível observar no Gráfico 2, as imagens agradáveis são alvo de uma avaliação da activação menos intensa, enquanto que as imagens desagradáveis provocam, segundo a avaliação dos participantes, uma activação mais elevada. Também se verificou significativa a interacção entre Grupo 
e Categoria, $F(6,207)=3,26, p<0,01$. Análises univariadas para cada categoria das imagens revelaram diferenças entre os grupos nas caras alegres, $F(2,72)=3,29$, $\mathrm{p}<0,05$, nas caras zangadas, $\mathrm{F}(2,72)=4,94, \mathrm{p}<0,01$, e nas imagens de guerra, $\mathrm{F}(2$, $72)=5,22, p<0,01$ (ver Gráfico 2). Pelo teste Tukey constata-se que o Grupo PTSD avalia as caras alegres e zangadas como mais activadoras que os Grupos NCA e NCB. Em relação às imagens de guerra, foram significativas as diferenças entre os Grupo NCB e os outros dois grupos (NCA e PTSD), sendo que o grupo NCB avalia este tipo de imagens como menos activadoras que os outros dois grupos.

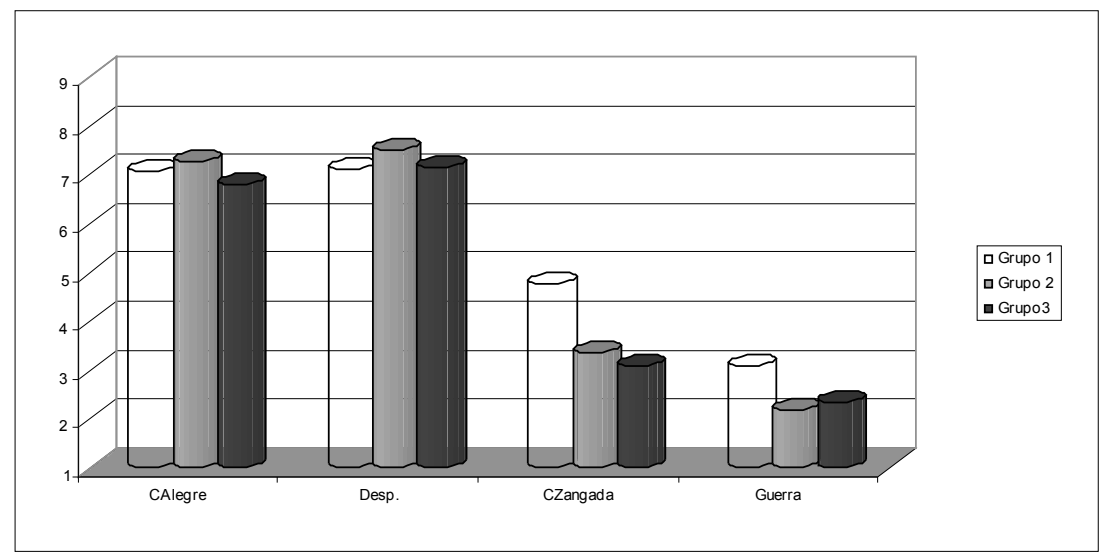

Gráfico 1. Avaliação média das 4 categorias de estímulos (caras alegres, desporto, caras zangadas e guerra), na escala SAM-Valência afectiva, para os três Grupos formados a partir da escala PCL-M (Grupo 1 - grupo NCB, Grupo 2 - grupo NCA, Grupo 3 - grupo PTSD).

Relativamente aos resultados obtidos na dimensão Dominância do SAM os valores remetem para um efeito principal do Grupo, $F(2,69)=6,72, p<0,001$. A análise post-hoc de Tukey revelou que o Grupo PTSD considera, no geral, as imagens como mais dominantes que o Grupo NCB. Também se obteve um efeito principal da Categoria, $F(3,207)=156,00, p<0,001$. A observação do Gráfico 3, permite verificar que as imagens agradáveis (caras alegres e desporto) são alvo de uma avaliação da dominância mais elevada do que as imagens desagradáveis. O efeito de interacção entre Grupo e Categoria também foi estatisticamente significativo, $F(6,207)=3,28, p<0,01$. Através de análises univariadas feitas para cada categoria das imagens, é possível verificar que existem diferenças entre os grupos no que se refere às caras zangadas, $F(2,72)=5,80, p<0,01$, e às imagens de guerra, $F(2$, $72)=6,77, p<0,01$. Isto é, as avaliações do Grupo NCB remetem para uma maior dominância dos estímulos que as dos Grupos NCA e PTSD. Através de testes Tukey, foi possivel observar diferenças significativas entre o Grupo NCB e os outros dois grupos para as caras zangadas e para as imagens de guerra. 


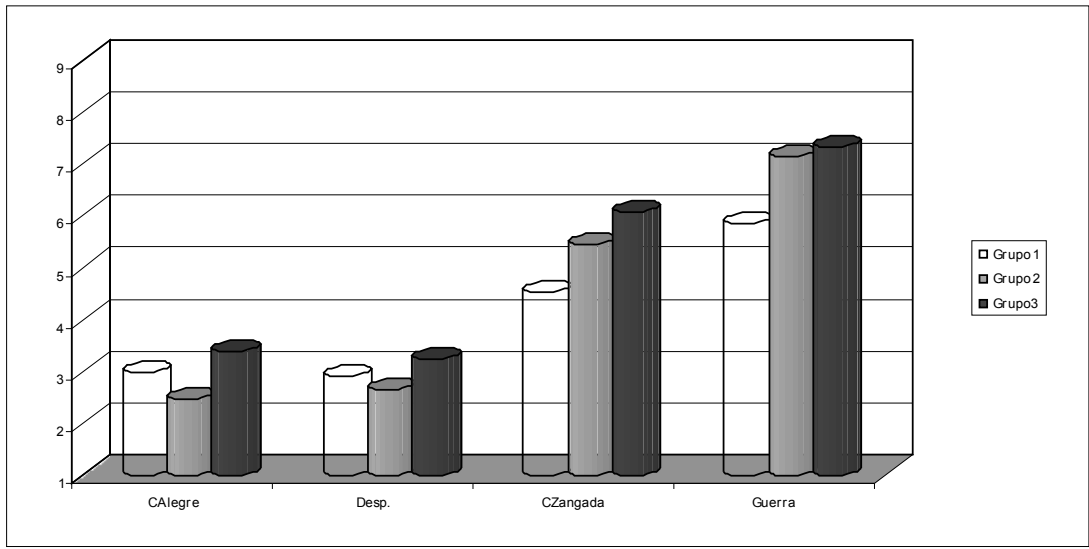

Gráfico 2. Avaliação média das 4 categorias de estímulos (caras alegres, desporto, caras zangadas e guerra), na escala SAM-Activação, em função dos três Grupos formados a partir da escala PCL-M (Grupo 1 - grupo NCB, Grupo 2 - grupo NCA, Grupo 3 - grupo PTSD).

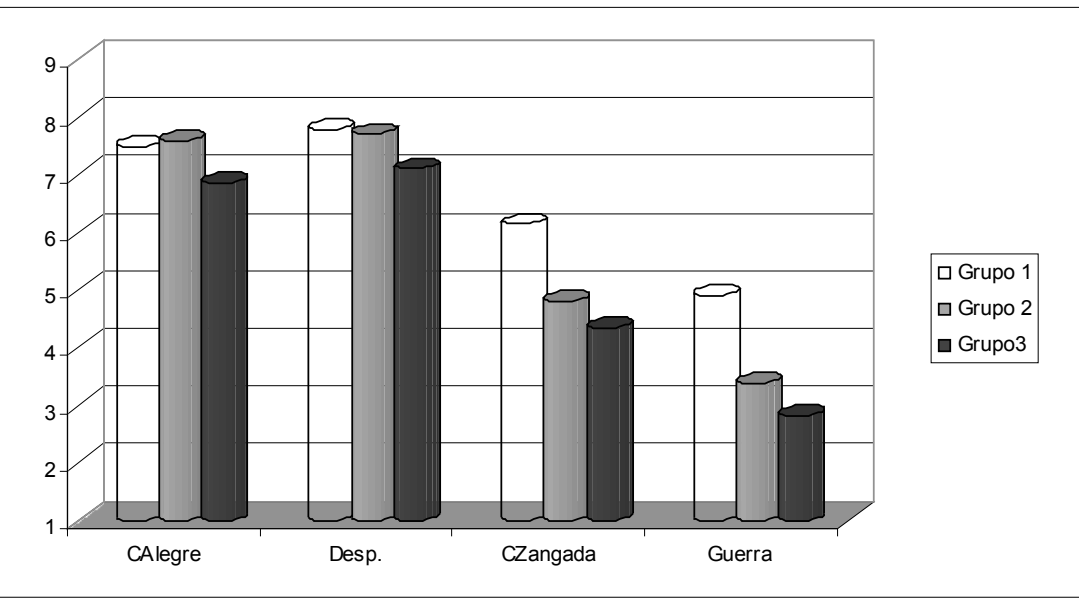

Gráfico 3. Avaliação média das 4 categorias de estímulos (caras alegres, desporto, caras zangadas e guerra), na escala SAM - Dominância afectiva, para os três Grupos formados a partir da escala PCL-M (Grupo 1 - grupo NCB, Grupo 2 - grupo NCA, Grupo 3 - grupo PTSD).

\section{Comparação das avaliações das imagens emocionais, entre os três gru- pos constituídos através da IES}

Constituindo os grupos a partir da escala IES, a distribuição dos participantes pelos grupos 1 e 2 foi um pouco diferente (ver Tabela 1) pelo que foram feitas análises de variância idênticas às ANOVAS apresentadas para os grupos constituídos a partir da PCL-M para cada dimensão do SAM. De uma maneira geral os resultados replicaram os efeitos obtidos com os grupos constituídos a partir do PCL-M, pelo que não são aqui apresentados os valores da análise. 


\section{Comparação das avaliações das imagens emocionais em função da escolaridade e do local onde estiveram na guerra}

Com o intuito de verificar se, para além da PTSD, existiam outras variáveis que influíam na avaliação dos estímulos, foi efectuada uma ANOVA $4 \times 2$ para cada uma das dimensões do SAM, com as variáveis Categorias das Imagens e Escolaridade (grupo com estudos até à $4^{\mathrm{a} C l a s s e, ~ e ~ g r u p o ~ c o m ~ e s t u d o s ~ s u p e r i o r e s ~ a ̀ ~} 4^{\mathrm{a}}$ Classe). Verificaram-se interacções significativas entre a categoria das imagens e os grupos para as três dimensões do SAM, Fs $(3,210)=3,48,3,55$, e 2,92, p<o,05, respectivamente para a Valência, Activação e Dominância. Os resultados permitem perceber que os sujeitos com menos anos de estudo utilizam mais os extremos das escalas do SAM, enquanto que aqueles que têm mais anos de escolaridade fazem uma utilização menos extremista das mesmas. No Gráfico 4 pode observar-se este efeito para a dimensão Valência.

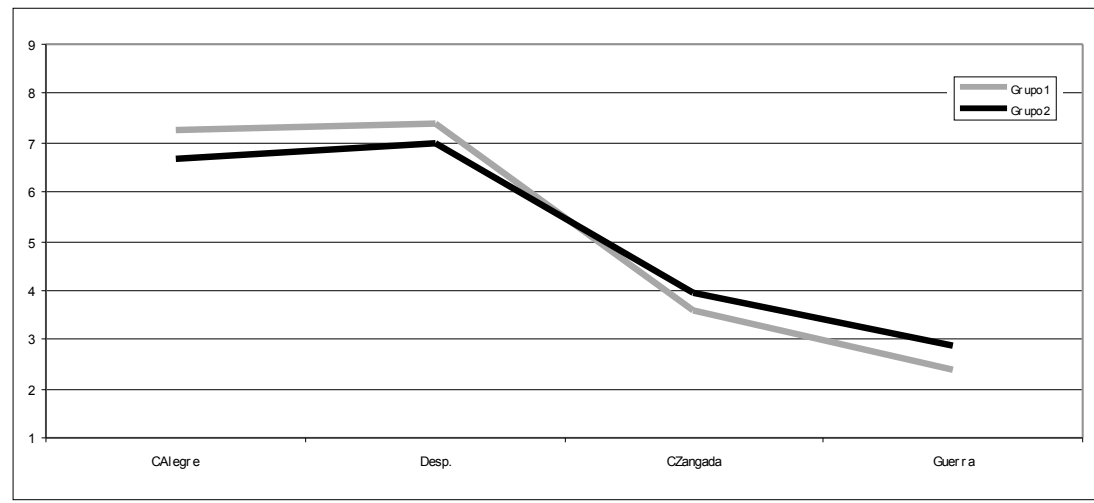

Gráfico 4. Avaliação média das 4 categorias de imagens (caras alegres, imagens de desporto, caras zangadas e imagens de guerra), para os dois grupos (Grupo 1-com escolaridade até

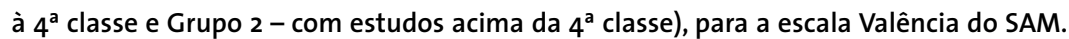

Avaliaram-se, ainda, os possiveis efeitos do local onde combateram, através de uma ANOVA $4 \times 3$, com as variáveis Categorias das Imagens e Localidade, sendo que se teve, apenas, em consideração os três locais principais (Angola, Moçambique e Guiné), ou seja, os palcos de guerra em que estiveram mais sujeitos. No entanto não se verificaram diferenças significativas entre os grupos para qualquer das dimensões do SAM.

\section{Discussão}

No presente estudo, foi examinada a avaliação de estímulos visuais em ex-combatentes com PTSD e em ex-combatentes sem PTSD. Os resultados obtidos remetem 
para uma avaliação dos estímulos desagradáveis por parte dos participantes com PTSD, ou sem diagnóstico mas com valores elevados nas escalas de auto-relato (Grupo NCA), mais negativa do que aquela que é feita pelos participantes sem sintomas de PTSD (Grupo NCB). No que respeita aos estímulos agradáveis, os resultados remetem para uma avaliação positiva dos mesmos por todos os grupos. Estes resultados vêm confirmar a hipótese de que existem diferenças na avaliação emocional das imagens em função da PTSD, no entanto infirmam a hipótese que sustenta que sujeitos com níveis de PTSD mais elevados possuem dificuldades em avaliar de forma favorável imagens agradáveis.

No que respeita aos estímulos desagradáveis, podemos verificar que o grupo com valores baixos nas escalas de PTSD, no geral, os avalia de forma menos negativa do que os grupos com sintomas de PTSD (com ou sem diagnóstico). Além disso, também em relação às outras dimensões, activação e dominância, se verifica, no geral, um padrão de maior diferenciação entre o grupo sem sintomas de PTSD e os grupos com sintomas (tendo ou não o diagnóstico). Assim, os indivíduos dos grupos com sintomas de PTSD avaliam os estímulos negativos com sendo mais activadores e associados a um menor controlo (valores baixos na dominância). Globalmente, estes resultados vão de encontro à hipótese prevista de que os sujeitos com PTSD avaliam de forma mais desfavorável as imagens de conteúdo desagradável, quer sejam imagens relacionadas com a própria guerra quer sejam rostos de pessoas.

A análise dos resultados relativos aos estímulos agradáveis releva o facto de todos os grupos avaliarem os mesmos de forma positiva. Apesar de existirem pequenas diferenças nas avaliações dos três grupos, o padrão geral é bastante semelhante na forma como são avaliadas as imagens de desporto e as caras alegres. Estes resultados não vêm portanto confirmar os estudos que sugerem que a PTSD interfere no normal processo de avaliação de estímulos agradáveis, sugerindo uma maior dificuldade em avaliar favoravelmente os estímulos de conteúdo positivo (Litz, 1992; Litz e Gray, 2002). Esta ausência de diferenças significativas sugere a possibilidade de existir uma inibição exógena da capacidade de reacção, dado determinados indivíduos estarem sujeitos à administração de fármacos. Observando as médias nos três grupos, verifica-se que os resultados obtidos vão no sentido do que Litz e Gray (2002) têm verificado nos seus estudos, sendo certo, porém, que estes autores defendem que, apesar de algumas vezes ser necessária uma estimulação positiva mais intensa, os ex-combatentes têm a capacidade de resposta a qualquer estado emocional.

Buckley, Blanchard, Neill (2000) sugerem que, em relação aos estímulos negativos, pessoas diagnosticadas com perturbações de ansiedade processam informação negativa de forma mais repentina do que sujeitos sem quaisquer perturbações desta ordem. Segundo Litz e colaboradores (1996), tal verifica-se, porque os indivíduos ansiosos tendem a estar mais atentos a estímulos ameaçadores. No presente 
estudo, verifica-se, factualmente, uma avaliação da activação superior nos grupos com sintomas de PTSD, que se refere às imagens de valência negativa, algo que remete para uma menor dominância e para uma avaliação mais negativa das mesmas. A análise ora desenvolvida é ademais, indicadora de que sujeitos com PTSD acentuada, quando expostos a estímulos negativos, são mais reactivos. Tal, pode dever-se a uma re-experiênciação, ainda que mental, do trauma, de acordo com o defendido por Foa e Hearst-Ikeda (1996). Pese embora o facto de, com o decorrer do tempo, a frequência e a intensidade das revivências ou reexperiências emocionais do trauma poderem vir a diminuir, geralmente, nunca desaparecerem completamente.

Litz e colaboradores (2000) defendem que quando a indivíduos com PTSD, é relembrado o seu trauma, estes podem, por um lado, desenvolver uma reacção emocional intensa ou, por outro lado, revelar desinteresse em circunstâncias em que, supostamente, deveriam demonstrar emoções.

No que diz respeito à escolaridade, o que se pôde verificar foi que esta influencia a forma como a escala é utilizada, sendo que os indivíduos com menos anos de estudo fazem uma utilização mais extremista do que aqueles com mais anos de estudo, contudo, e porque existem indivíduos com escolaridade mais baixa e mais elevada nos três grupos, pensamos que estas diferenças na utilização da escala não terão afectado os resultados apresentados.

Relativamente ao Local ou Palco de Guerra, não se verificou uma influência significativa na avaliação das imagens. Embora se pudesse supor que os sujeitos que estiveram na Guiné, por ser geralmente reconhecido como o pior local, fizessem uma avaliação mais desfavorável de qualquer tipo de estímulos, em particular de imagens de guerra, esse facto não se verificou.

Apesar dos resultados, globalmente, mostrarem diferenças na avaliação dos estímulos emocionais de acordo com o esperado, surgem algumas limitações no presente trabalho. As medidas de auto-relato têm algumas questões associadas, como a tão sobejamente conhecida desejabilidade social, uma vez que em contextos em que os participantes sentem que estão a ser avaliados poderá existir a tendência para responder de acordo com pensam ser o mais adequado socialmente. Neste contexto, o complemento destas respostas com algum tipo de indicador psicofisiológico, como a frequência cardíaca, respiratória ou a actividade electrodérmica, teria sido útil.

No caso das escalas utilizadas, IES e PCL-M, nenhuma delas se baseia em indicadores de validade psicométrica para a população portuguesa. Contudo é de notar que a diferenciação entre o grupo com diagnóstico de PTSD e os outros dois nas escalas de auto-relato vem, em parte, validar a própria escala. Também os resultados, ao diferenciarem as avaliações das imagens feitas pelo grupo com 
valores baixos dos dois grupos com valores elevados nas escalas, vêm mostrar a validade discriminante das escalas utilizadas.

Em todo o caso, a investigação científica desenvolvida na área das respostas negativas em indivíduos com PTSD tem sido considerável. Contudo, no que concerne à investigação sistemática sobre os défices emocionais de resposta na PTSD, bem como à relação e interacção entre estas duas classes de respostas, a investigação tem sido escassa.

O entorpecimento emocional é representado em três dos critérios de diagnóstico da PTSD na DSM-IV-R (APA, 2002), a saber: perda do interesse ou participação em actividades significativas ( $C_{4}$ ); sentir-se distante ou desligado em relação aos outros ( $C$ 5); gama de afectos restringida ( $C$ 6). No entanto, este conceito ainda é alvo de grandes dúvidas, pelo que se torna pertinente perceber a forma como são processadas as emoções em indivíduos com PTSD.

Em investigações futuras sugere-se, uma vez que as emoções são determinadas por inúmeros factores, a utilização de outros tipos de apresentação de estímulos (ex. filmes) e outras formas de estimulação (ex. auditivos). Pensa-se que seria, ainda, pertinente complementar a avaliação verbal com registos psicofisiológicos, tanto a nível do Sistema Nervoso Autónomo como do Sistema Nervoso Central, bem como a actividade motora da expressão facial (ver Ekman et al., 1983) para uma investigação mais aprofundada neste campo.

\section{Referências bibliográficas}

Albuquerque A., Fernandes A., Saraiva E., Lopes F. (1992). Distúrbios Pós-Traumáticos do Stress em Ex-Combatentes da Guerra Colonial. Revista de Psicologia Militar, 1-9.

American Psyshiatric Association (2002). DSM-IV-TR: Manual de Diagnóstico e Estatística das Perturbações Mentais, $4^{\mathrm{a} E d}$. (Fernandes C., Figueira M., Sampaio D. \& Almeida J.), Climepsi Editores, Lisboa. (Obra original publicada em 2000).

Anunciação C. (2004). A Perturbação Pós-Stress Traumático em Militares e Civis nas Zonas de Conflito. Revista Portuguesa de Saúde Militar, Ano V, $\mathrm{N}^{\circ} 1$.

Buckley T., Blanchard E., Neill T (2000). Information processing and PTSD: a review of the empirical literature. Clinical Psychology Reviem, Vol. 28, 8, p. 1041-1065.

Cacioppo J., Gardner W., (1999). Emotion, Annual Review Psychology, 50, 191-214.

Damásio A., (2001). O Sentimento de Si, O corpo, a Emoção e a Neurologia da Consciência, $13^{\text {a }}$ Edição, Publicações Europa-América, Lisboa.

Engdahe B., Dikel T., Ebery R., Blank A. (1997). Posttraumatic Stess Disorder in a Community Sample of Former Prisioners of War: A Normative Response to Severe Trauma. American Journal of Psychiatry, 154/11, 1576-1581.

Ekman, P. \& Friesen, W. V. (1975). Unmasking the face. A guide to recognizing emotions from facial clues. Englewood Cliffs, New Jersey: Prentice-Hall.

Ekman, P., Levenson, R. W., Friesen, W. V. (1983). Emotions differ in autonomic nervous system activity. Science, 221, 1208-1210. 
Foa E., Hearst-Ikeda D. (1996). Emotional Dissociation in Response to Trauma An Information - Processing Approach. Handbook of Dissociation: Theoretical, Empirical, and Clinical Perspectives. Editado por Larry K. Michelson e William J. Ray. Plenum Press, New York, 207-224.

Foa E., Keane T., Friedman M. (2000). Guildelines for treatment of PTSD. Journal of Traumatic Stress, Vol. 13, $\mathrm{N}^{\circ} 4$.

Friedman M. J., Yehuda R. (1995). Post-Traumatic stress disorder and comorbidity: psychobiological approaches to differential diagnosis. In: Friedman M.J., Charney D.S., Deutch AY. Neurobiological and clinical consequences of stress. Philadelphia: LippincottRaven; p. 429-45.

Friedman M. J. (1997). Posttraumatic Stress Disorder. The Journal of Clinical Psychiatry, Vol.58, Suplemento 9, p.33-36.

Golier J, Yehuda R, and Southwick S. (1997). Memory and Posttraumatic Stress Disorder. Em P.S. Appelbaum, L.A. Uyehara, e M.R. Elin (Eds.) Trauma and Memory Clinical and Legal Controversies. Cap. 8, p. 225-242.

Goodwin J. (1987). The Etiology of Combat-Related Post-Traumatic Stress Disorders. Tom Williams (Ed). Post-Traumatic Stress Disorders: a handbook for clinicians, p.1-18.

Halligan S., Yehuda R,, (2000). Risk Factors For PTSD. The National Center for Post-Traumatic Stress Disorder, PTSD Research Quarterly, volume II, número 3.

Holmes E. (2003/2004). Intrusive, Emotional Mental Imagery and Trauma: Experimental and Clinical Clues. Imagination, Cognition and Personality, Vol. 23, Número 2-3, p.147-154.

Horowitz M., Wilner M., Alvarez W., (1997). Impact of Event Scale: A measure of subjective stress, Psychosomatic Medicine, 41, 209-218.

Keane T. (1993). Symptomatology of Vietnam Veterans whith Posttraumatic Stess Disorder. Posttraumatic Stress Disorder DSM-IV and Beyand, Cap. 6. American Psychiatric Press, 99-110.

Keane, T., Street, A., Orcutt, H.. (2000). Posttraumatic stress disorder. Editado em M. Hersen \& M. Biaggio (Eds.), Effective brief therapy: A clinician's guide p. 139-155. San Diego, CA: Academic Press.

Lang, P. J. (1980). Behavioral treatment and bio-behavioral assessment: computer applications. In J. B. Sidowski, J. H. Johnson, \& T. A. Williams (Eds.), Technology in mental health care delivery systems (pp. 119-137). Norwood, NJ: Ablex.

Lang P., Bradley M., Cuthbert B., (1997). International affective picture system (IAPS): Technical Manual and Affective Ratings, Center for the Study os Emotiion and Attention.

Litz, B.T. (1992). Emotional numbing in combat-related post-traumatic stress disorder: A critical review and reformulation. Clinical Psychology Review, 12, 417-432.

Litz, B., \& Gray, M. (2002). Emotional numbing in posttraumatic stress disorder: Current and future research directions. Australian and New Zealand Journal of Psychiatry, 36, 198-204.

Litz B., Orsillos S., Koloupek D., Weathers F. (2000). Emotional Processing in Posttraumatic Stress Disorder. Journal of Abnormal Psychology, Vol.109, № ${ }^{1}$, p.26-39.

Lundqvist, D., Flykt, A., \& Öhman, A. (1998). The Karolinska Directed Emotional Faces. Stockholm: Karolinska Institute, Psychology section, Department of Clinical Neuroscience.

van der Kolk, B.A. (2001). The Assessment and Treatment of Complex PTSD. Editado por Rachel Yehuda, ed. American Psychiatric Press.

Weathers F., Litz B., Herman D., Huska J., Keane T., (1993) The PTSD Checklist (PCL): Rlialibity, Validity, and Diagnostic Utility, Annual Meeting of International Society for Traumatic Stress Studies, San Antonio, TX. 


\section{L'évaluation de stimuli émotionnels dans ex-combattants de guerre avec et sans PTSD}

Cette étude visait à étudier comment les images émotionnelles son évalués par ex-combattants de guerre. L'étude a été menée auprès d'un échantillon de 72 sujets qui ont été soumis à deux échelles d'évaluation du PTSD et à l'affichage et la catégorisation d'images avec le Self-Assessement Manikin (SAM; Lang, Bradley \& Cuthbert, 1997).

Nous avons comparé trois groupes, un avec diagnostic de PTSD (PTSD groupe) et deux sans un diagnostic de PTSD, fondés sur les échelles d'auto-évaluation: un avec des valeurs faibles (Groupe Non-Glinique Basse - NCB) et un autre avec des valeurs plus élevés (Groupe Non-Clinique de Haut - NCA). On a constaté que le groupe de PTSD et le groupe NCA évaluent plus négativement les images désagréables (images de guerre et de 'visages menaçants') par rapport au groupe NCB. De plus, dans l'ensemble, les mêmes groupes évaluent aussi les images désagréables come plus activateurs et dominantes, en comparaison au groupe NCB. En ce qui concerne les images agréables, la différenciation entre les trois groupes n'a pas été pas significative.

MOTS-CLÉS: Emotion; PTSD; Engourdissement émotionnel; Combattants de guerre.

\section{Evaluation of emotional stimuli in war soldiers with and without PTSD}

The present study has been carried out to inquire how emotional images are evaluated by former war soldiers who were actively involved in the Portuguese colonial war. The study was produced with 72 subjects who had been submitted not only to two different posttraumatic stress disorder (PTSD) assessment scales, but also to the image visualization and categorization through the Self-Assessment Manikin (SAM; Lang, Bradley \& Cuthbert, 1997).

Three groups of subjects were studied and compared: one group of subjects with PTSD, and two groups of subjects without PTSD, grouped according the self-assessment scales: one with lower levels (non-clinical low group - NCB), and another with higher levels (non-clinical high group - NCA). Both PTSD and NCA groups evaluate more negatively the unpleasant images showed (war images and "disgusting faces") in comparison to the NCB group. Beyond that, mainly, the same groups, also evaluate unpleasant images as much activating and dominant than the NCB group. Concerning to the pleasant images, there was no significant difference between the three groups of subjects.

KEY-WORDS: Emotion; PTSD; Emotional numbing; War soldiers. 